\title{
Three novel mutations of the fibrillin-1 gene and ten single nucleotide polymorphisms of the fibrillin-3 gene in Marfan syndrome patients
}

Received: 16 February 2004/ Accepted: 27 April 2004/Published online: 23 June 2004

(C) The Japan Society of Human Genetics and Springer-Verlag 2004

\begin{abstract}
Marfan syndrome (MFS) is an autosomal dominant disorder of the extracellular matrix. Allelic variations in the gene for fibrillin-1 ( $F B N 1)$ have been shown to cause MFS. To date, over 550 mutations have been identified in patients with MFS and related connective tissue diseases. However, about a half of MFS cases do not possess mutations in the FBN1 gene. These findings raise the possibility that variants located in other genes cause or modify MFS. To explore this possibility, firstly we analyzed $F B N 1$ allelic variants in 12 Japanese patients with MFS, and secondly we analyzed fibrillin-3 gene $(F B N 3)$ in patients without $F B N 1$ mutations using conformation sensitive gel electrophoresis (CSGE) and direct sequencing analysis. We identified three novel FBN1 mutations and ten FBN3 single nucleotide polymorphisms (SNPs). In this report, we could not detect a responsible mutation of the $F B N 3$ gene for MFS. Although the number of the cases in this report is small, at least these results suggest that diseasecausing mutations in exon regions of the $F B N 3$ gene are very rare in MFS.
\end{abstract}

Keywords Marfan syndrome - Fibrillin-3 - FBN3 • Fibrillin-1 $\cdot F B N 1 \cdot$ Connective tissue disorder

Nucleotide sequence data reported are available in the DDBJ/ EMBL/GenBank databases under the accession numbers: AB177797, AB177798, AB177799, AB177800, AB177801, $\mathrm{AB} 177802, \mathrm{AB} 177803$

T. Uyeda $\cdot$ T. Takahashi $\cdot$ S. Eto $\cdot$ T. Sato $\cdot$ G. Xu

R. Kanezaki $\cdot$ T. Toki $\cdot$ E. Ito $(\square)$

Department of Pediatrics,

Hirosaki University School of Medicine,

5 Zaifu-cho, Hirosaki 036-8563, Japan

E-mail: eturou@cc.hirosaki-u.ac.jp

Tel.: + 81-172-395070

Fax: + 81-172-395071

S. Yonesaka

Hirosaki University School of Health Sciences,

66 Hon-cho, Hirosaki 036-8564, Japan

\section{Introduction}

Marfan syndrome (MFS; MIM \#154700) is an autosomal dominant disorder of connective tissue. The disease has an incidence of $1 / 5,000$ with over $25 \%$ of sporadic cases. In 1991, the fibrillin-1 gene (FBN1; MIM 134797) was identified as a major disease-causing gene of MFS (Lee et al. 1991). FBN1 on chromosome 15q21.1 codes for fibrillin-1, a main component of extracellular microfibrils. Since the identification of $F B N 1$, over 550 mutations have been identified (Collod-Beroud et al. 2003). Although the mutation detection rate varies from 23.5 to $80 \%$, the overall mutation detection rate is approximately $50 \%$ even in patients with classic MFS. FBN1 mutations have been also found in individuals who do not satisfy the diagnostic criteria for MFS such as autosomal dominant ectopia lentis (EL; MIM 129600). Therefore, the wide spectrum of variability of MFS is not wholly explained solely by $F B N 1$ mutations. It is possible that another unknown candidate gene or genes causing or modifying the disease might exist. For example, the fibrillin-2 gene ( $F B N 2$; MIM 121050) on human chromosome 5q23-q31 shares a high degree of homology with FBNl and has been known to cause congenital contractural arachnodactyly (CCA; MIM 121050) (Lee et al. 1991). The fibrillin-3 gene (FBN3) on human chromosome 19p13 also has high homology to other fibrillin family members (Nagase et al. 2001). The $F B N 3$ gene is fragmented into 63 exons, transcribed in a $9 \mathrm{~kb}$ mRNA that encodes a 2,809 amino acid protein and has overall homology of greater than $60 \%$ with either $F B N 1$ or $F B N 2$, and contains multiple EGF-like domains. Expression of $F B N 3$ is highest in brain tissue.

The spectrum of overlapping disorders presenting with FBN1 mutations like EL define the molecular group of type 1 fibrillinopathies (Collod-Beroud and Boileau 2002). Weill-Marchesani syndrome (WMS; MIM 277600) is also a connective tissue disorder characterized by short stature, brachydactyly, joint stiffness, and eye anomalies including ectopia lentis. 
Fibrillin immunofluorescence studies of skin biopsies from the patients with WMS show a decrease in immunostainable fibers compared to unaffected controls. Linkage analysis has mapped the autosomal dominant (AD) WMS locus to chromosome 15q21.1 (Wirtz et al. 1996). The FBN1 gene was sequenced in an AD WMS family and in flame deletion of 24 nucleotides was found (Faivre et al. 2003a). These studies suggest that WMS is an allelic condition with MFS and one of a type 1 fibrillinopathies. Interestingly, the autosomal recessive (AR) WMS locus has recently been mapped to chromosome 19p13.3-p13.2, where the FBN3 gene is located (Faivre et al. 2003b), suggesting that $F B N 3$ is a candidate gene for MFS as well as AR WMS. We analyzed allelic variants of the $F B N 3$ gene as well as $F B N 1$ gene using conformational sensitive gel electrophoresis (CSGE) and DNA sequencing in Japanese patients with MFS.

\section{Materials and methods}

Genomic DNA extraction and polymerase chain reaction (PCR) amplification

Genomic DNA was collected from 12 patients of ten families containing two couples of parent-child that fulfilled the Ghent nosology revised diagnostic criteria (De Paepe et al. 1996) for the diagnosis of MFS and 50 Japanese volunteers who served as normal controls. Written informed consent for genomic examination was obtained from all participants and the Ethic Committee of Hirosaki University School of Medicine approved this study. Genomic DNA was extracted from peripheral blood leukocytes by the QIA amp DNA blood mini kit (Qiagen, Inc.). PCR primers used to amplify the individual exons of $F B N 1$ were derived from previously reported information (Korkko et al. 2002). PCR primers of $F B N 3$ on Table 1 were originally designed. PCR amplification was performed using $40 \mathrm{ng}$ of genomic DNA, $0.25 \mathrm{mmol} / 1$ of forward and reverse primers, $2 \mathrm{mmol} / 1 \mathrm{MgCl}_{2}, 0.2 \mathrm{mmol} / 1 \mathrm{dNTPs}$, and $1 \mathrm{U}$ of Taq DNA polymerase. The PCR conditions consisted of initial denaturation at $95^{\circ} \mathrm{C}$ for $10 \mathrm{~min}$, followed by 35 cycles at $95^{\circ} \mathrm{C}$ for $25 \mathrm{~s}$, $58-68^{\circ} \mathrm{C}$ for $25 \mathrm{~s}$, and $72^{\circ} \mathrm{C}$ for $35 \mathrm{~s}$, with a final extension at $72^{\circ} \mathrm{C}$ for $10 \mathrm{~min}$.

Heteroduplex analysis and sequencing

Heteroduplex analysis was performed using CSGE. CSGE was performed essentially using the same conditions as previously described (Ganguly et al. 1993; Korkko et al. 1998). Samples with abnormal CSGE band patterns were directly sequenced in both directions by means of the ABI PRISM Bigdye Terminator Cycle Sequencing Ready Reaction Kit
(Applied Biosystems). Forward and reverse primers were the same as those used for amplification.

Population studies

One hundred chromosomes from 50 unrelated controls were tested for the identified mutations by using restriction enzyme digestion or direct sequencing to determine recurrent mutations or polymorphisms, and to confirm their association with the pathologic condition under study.

\section{Results}

First, we screened $F B N 1$ allelic variants in MFS patients and identified three novel mutations in four MFS patients containing a parent-child couple. These three mutations consisted of the two nonsense variants and one missense variant shown in Table 2 . These variants were completely absent from the genomes of 50 normal control samples. The missense mutation on exon 63 (c. $8121 \mathrm{G}>\mathrm{C}$, p. Cys 2663 Ser) was detected in a parentchild couple. Although we tried to analyze the mutations of other family members, we could not obtain informed consent from them.

Next, we identified ten $F B N 3$ allelic variants in MFS patients without $F B N 1$ mutation using CSGE and sequence analysis. All of ten variants were analyzed by restriction enzyme digestion or direct sequencing and compared to 50 normal control samples. All variants of the FBN3 gene were also detected in normal controls in either homozygous or heterozygous status (Table 2).

\section{Discussion}

Although mutations of the FBN1 gene cause MFS, the disease-causing genes remain unknown in about half of MFS patients. In this study, mutations of the FBNI gene were detected in only four of 12 patients with MFS, even using very sensitive CSGE and sequence analysis. In order to investigate the possibility that a portion of MFS might be caused by mutation of another fibrillin gene, we screened mutations of the $F B N 3$ gene and identified multiple allelic variants. However, a responsible gene mutation for MFS was not detected in these patients. Although the number of the cases included in the present study was small, these results suggest that disease-causing mutations in exon regions of the $F B N 3$ gene are either very rare in MFS or that the $F B N 3$ gene is not a responsible gene for MFS. Functional variant analysis of the $F B N 3$ gene or more widespread screening of $F B N 3$ gene variants might determine a relationship between $F B N 3$ and several connective tissue disorders. 
Table 1 Primer sequences

\begin{tabular}{|c|c|c|c|}
\hline Exon & Sense primer & Antisense primer & $\begin{array}{l}\text { Product } \\
\text { size (bp) }\end{array}$ \\
\hline 1 & 5'-GCCTCCTCTGACTGTTCACC & 5'-ACAGCCCAATCCACACCGAA & 405 \\
\hline 2 & 5'-GCAGCCCAGGCATCTTGCAG & 5'-CCTGGCTAAGTCCCCGTGTAAAC & 329 \\
\hline 3 & 5'-AGACCCCTTCGGTGTGGATTGG & 5'-ACAGGAAGGCAGGACGCATAGTAA & 345 \\
\hline 4 & 5'-ATTACTATGCGTCCTGCCTTCCT & 5'-AAGAGACTGTGGCAATAAACGTCC & 271 \\
\hline 5 & 5'-ССТСАGTCТTАТTCAАССТGGGCC & 5'-TCCATCGAGTCCCCTGTCTACCT & 262 \\
\hline 6 & 5'-CGCTGAGAAGGTGTTAGAGACGCA & 5'-GGAGGGAAGAACCAAGGCAGCTAA & 346 \\
\hline 7 & 5'-CTTAGCTGCCTTGGTTCTTCCCTC & 5'-TCCAGTCACCCACCTTCACATGC & 203 \\
\hline 8 & 5'-AGGTGTGAGCTACTGCATCTGGAC & 5'-GCAGGCTGCAGCTCTTACTCAC & 369 \\
\hline 9 & 5'-GCCTCAACCCTGCTTCCTTG & 5'-ATCCTTCAAGTCTTGGAATGTTCCT & 250 \\
\hline 10 & 5'-TCCATGAGAAAGAGGGCAGGATC & 5'-GGTGCATTCGTCTACATCTGAGG & 360 \\
\hline 11 & 5'-TCTGCCGACACTTCACCAACCTGT & 5'-ССССААСААСАТСАССССТАСТСТ & 353 \\
\hline 12 & 5'-ATCCAAGAGTAGGGGTGATG & 5'-GGCACAGTGAAGATTTGAGGT & 300 \\
\hline 13 & 5'-GGACGGACTAATATACAAGCTCAC & 5'-TCTTCTCCTTCTCTCATGCTCGT & 406 \\
\hline 14 & 5'-GAGGACAGAGACACAGACAACAGG & 5'-AGGCATTCAGACCAAGGAGGCGA & 481 \\
\hline 15 & 5'-CGGGGTTGGAGTTGTGTCTTAGTC & 5'-ААСТССТАСАGССТСССАССАСА & 266 \\
\hline 16 & 5'-CTCGGACACACCСТCСТTCT & 5'-GGGCACACTCATCCACGTCT & 282 \\
\hline 17 & 5'-ACCGCTGTGTCTGCAACCTG & 5'-CTTTGAGCGTGTGCATGCCT & 348 \\
\hline 18 & 5'-AGTGCCCTCTGCATGCTTGT & 5'-ACCTGCACCCTGACCCATAG & 300 \\
\hline 19 & 5'-GCTGGACCCCTCTGGTACCT & 5'-CCCCATGGAGGGCTTTTCCCA & 328 \\
\hline 20 & 5'-ТСТСТТСАСТСТTGTTСТСССAGT & 5'-TCACACTCGTTCACATCTGGGTA & 375 \\
\hline 21 & 5'-CTCCTTACCCAGATGTGAACGA & 5'-GGGAACAAAGGAAGAACGTG & 190 \\
\hline 22 & 5'-CTGGCCCTCGACCTGACTAC & 5'-TTCTTAGTGCCTCGCCTTACCCA & 470 \\
\hline 23 & 5'-TGGTTTGATGCCCCTGTACTTCA & 5'-TGTCTCTACGTCTTATTTGAGGCC & 225 \\
\hline 24 & 5'-ССТСТАGСССАСАССТССАТСААА & $5^{\prime}$-АTCCACGTCCACACAGCAACAGC & 305 \\
\hline 25 & 5'-CCAGCATGAGCCTCATCACC & 5'-САСАТТСАССАGАСАТССАСТСАС & 278 \\
\hline 26 & 5'-GGGTGTGTATATGGGTGTCTGTGC & 5'-TGGGAGCAGAGTGGAGTTGAGGT & 430 \\
\hline 27 & 5'-TGCATCCTGCAGACATCAACGAATG & 5'-GGCTGGCTTCACTATGCTGT & 242 \\
\hline 28 & 5'-AGTGACCATCGCTATCCTCACCT & 5'-AATTCTGACACTGCCTCCTCCAC & 297 \\
\hline 29 & 5'-CTCAGACCTCATGCTCTCTTC & 5'-GCACTGTATAGGACTCTGTTCTTG & 298 \\
\hline 30 & 5'-AGAGGAAGAGTCCСТGCССТTТC & 5'-ССССАССТАССТСТАСТGСТ & 253 \\
\hline 31 & 5'AGGGTTGGAGGGTTGATGGGTCT & 5'-AGAAGGAGTCAGGAGGTCGAGTG & 313 \\
\hline 32 & 5'-GAATCTTGGGGCCCCTCAGACA & 5'-TGCGCACACTCGTCCACATC & 255 \\
\hline 33 & 5'-AGAGGACAGAGTGGGTAGGTC & 5'-CATGTCCCCTGCCCCAACTC & 252 \\
\hline 34 & 5'-AGAACAGAGCAGGAGAGGGAATG & 5'-CAGGAGCAGGTAGATTTGAACACG & 428 \\
\hline 35 & 5'-GCTТTСССТСТGАСАССТАСТААС & 5'-ТCTTGGTTTTCСТGTCGCCTAAGC & 300 \\
\hline 36 & 5'-TCCTGCTGTTGCTCCССТGTTTC & 5'-GCATAGTGTGGTCCTGCTGCTCA & 298 \\
\hline 37 & 5'-GTCCTCTGCATGGGTTTAGCTCC & 5'-ССТТСССТССТСТАСССТТGTGA & 349 \\
\hline 38 & 5'-GAAGTGGCCTTGTGCTGGGTCTC & 5'-TGACTGCCAGTTCCCTAGGTCATC & 252 \\
\hline 39 & 5'-AGTTCCTATCTCTTTGGCTCCCT & 5'-АСТТСААСТСТСТGGСТСТСАТСА & 355 \\
\hline 40 & 5'-TAAGTGGAAGGAGGGAAAGGGTG & 5'-GAAAAGAGCTGTTGAGGAGGGGA & 291 \\
\hline 41 & 5'-GGCCACAACAGCCTCAACCA & 5'-GGGAGAGGTGGCACCTTCAC & 247 \\
\hline 42 & 5'-GCСТCСАТСТСАТАТААССАСССТ & 5'-GACAGAGCCATACCCCATCTAAGT & 349 \\
\hline 43 & 5'-TAGGATGTGATAGAGGTGAGAGCT & 5'-TGAACTAGGATGGACAGAAACACC & 393 \\
\hline 44 & 5'-GTAAATGGTTGATCTGCCTCCCTG & 5'-GGACTTTCTGGGCATCTTTCTGTG & 431 \\
\hline 45 & 5'-GTGGACAGAAGTACAAGGAAGGTT & 5'-GTAAATGGATCTATGCTCACCCAG & 277 \\
\hline 46 & 5'-ACAGTGATTGCAGGTGTTGGTCC & 5'-ТССАССТТСАТСАСААСССТАGC & 305 \\
\hline 47 & 5'-CCTGACCATGACCTTTACTCTGGA & 5'-ACACACCTAGCTCAGAATGGACC & 432 \\
\hline 48 & 5'-ATCAGCACAACCCATTCACCAGGA & 5'-TGTGCAGGGAAGGATGAGAGAAG & 340 \\
\hline 49 & 5'-ТСТСТCAТCСТTCССТGCACACA & 5'-CACACTCATTCACGTCTGCGGAT & 381 \\
\hline 50 & 5'-AGCСССТСАСССАСАТGCTAAGAG & 5'-GGGAGAGAGGCAGTGGTTAAGGAC & 475 \\
\hline 51 & 5'-GACTAGAGGTGCATTCGATCTGGC & 5'-GTATTGTGGCAGCGGAAGGCACA & 441 \\
\hline 52 & 5'-TTACTCCCACCCAGACACAGACG & 5'-CCAGGTTCAAGCGATTCTCCTGTC & 496 \\
\hline 53 & 5'-GAATCCAGGTCCCCACAGCCATA & 5'-ACTGCCTGTCCCTCCCATTCATTG & 383 \\
\hline 54 & 5'-ССССТСССАТСТCAGATGAC & 5'-CAGTGGGTGCCACATGGTAG & 198 \\
\hline 55 & 5'-AGTGACCGTTGTCTTTCCCA & 5'-AGACACATGACCCTGAGCAAAC & 310 \\
\hline 56 & 5'AGTGGCCGGTGTGGAAGGGA & 5'-GGCTCACCCAGGCAGGTAGT & 319 \\
\hline 57 & 5'-CTGCTTACCCTCAATGGCTGCTTT & 5'-AGAGGGGTGACAAGAGAAAGACCT & 492 \\
\hline 58 & 5'-TCCGAGGTCTTTCTCTTGTC & 5'-AAATAACTAATGCTGGGGTCATGG & 289 \\
\hline 59 & 5'-AGGCACAGAGAGGGTAGGTAAGT & 5'-AGAGAGAGGGAGAGAGGGTTTAC & 467 \\
\hline 60 & 5'-ACССТGCCTGATCTCТСТССТCТ & 5'-CATCATCTTGCAGGGTGGCGTCT & 401 \\
\hline 61 & 5'-TTGGCATTGGACTTGGGCTCTGAA & 5'-GCGGGGCATGGAGTCTGCTT & 389 \\
\hline 62 & 5'-ССТСТТТСССАСАСТСАСССАТТ & 5'-AGGGACACACATGCACACCCTTAT & 386 \\
\hline $63 \mathrm{~A}$ & 5'-AACTCTTGTCTCССТСССТСТСТC & 5'-CCAGGTGTGAGAGGTTCAGG & 397 \\
\hline $63 \mathrm{~B}$ & 5'-TGAACCTGGCCACCCTTGAC & 5'-GTCCTTCCCAGTTCCAGAATCC & 404 \\
\hline $63 \mathrm{C}$ & 5'-CTACCGGCTGGAGGTGGTGA & 5'-GCCTCTTCCTGACCTTGGCT & 384 \\
\hline $63 \mathrm{D}$ & 5'-GGAAGAGTGAAATGCTACAC & 5'-АCТTTATTATCGTCTCCACT & 396 \\
\hline $63 \mathrm{E}$ & 5'-GCCCAGACTCCACAGTGACTTG & 5'-AATTAGCTGGGTGTGGTGGCGTG & 430 \\
\hline
\end{tabular}


Table $2 F B N 1$ and $F B N 3$ allelic variants identified in 12 MFS patients and 50 normal controls

\begin{tabular}{|c|c|c|c|c|}
\hline Exon & Nucleotide change & Amino acid change & $\begin{array}{l}\text { Patient frequency } \\
\text { genotype (allele) }\end{array}$ & $\begin{array}{l}\text { Control frequency } \\
\text { genotype (allele) }\end{array}$ \\
\hline \multicolumn{5}{|c|}{ FBN1 allelic variants } \\
\hline 6 & c. $719 \mathrm{C}>\mathrm{T}$ & p.Gln $136 \mathrm{X}$ & $1 / 12(1 / 24)$ & $0 / 50(0 / 100)$ \\
\hline 33 & c. $4229 \mathrm{~T}>\mathrm{C}$ & p.Gln 1366 X & $1 / 12(1 / 24)$ & $0 / 50(0 / 100)$ \\
\hline 63 & c. $8121 \mathrm{G}>\mathrm{C}$ & p.Cys $2663 \mathrm{Ser}$ & $2 / 12(2 / 24)$ & $0 / 50(0 / 100)$ \\
\hline \multicolumn{5}{|c|}{$F B N 3$ allelic variants } \\
\hline 11 & c. $1833 \mathrm{G}>\mathrm{A}$ & p.Arg $473 \mathrm{Gln}$ & $3 / 12(3 / 24)$ & $7 / 50(14 / 100)$ \\
\hline 22 & c. $3219 \mathrm{G}>\mathrm{T}$ & p.Arg 935 Leu & $1 / 12(1 / 24)$ & $16 / 50(18 / 100)$ \\
\hline 22 & c. $3227 \mathrm{G}>\mathrm{T}$ & p.Val 938 Phe & $1 / 12(1 / 24)$ & $2 / 50(2 / 100)$ \\
\hline 25 & c. $3662 \mathrm{C}>\mathrm{T}$ & p.Arg 1083 Trp & $3 / 12(3 / 24)$ & $15 / 50(19 / 100)$ \\
\hline 30 & c. $4318 \mathrm{~A}>\mathrm{G}$ & p.Pro 1301 Pro & $3 / 12(3 / 24)$ & $45 / 50(71 / 100)$ \\
\hline 38 & c. $5255 \mathrm{G}>\mathrm{A}$ & p.Gly 1614 Ser & $2 / 12(2 / 24)$ & $1 / 50(1 / 100)$ \\
\hline 44 & c. $6022 \mathrm{C}>\mathrm{G}$ & p.Asn 1869 Lys & $2 / 12(2 / 24)$ & $15 / 50(19 / 100)$ \\
\hline 45 & c. $6125 \mathrm{C}>\mathrm{T}$ & p.Leu 1911 Phe & $2 / 12(2 / 24)$ & $18 / 50(21 / 100)$ \\
\hline 48 & c. $6592 \mathrm{G}>\mathrm{A}$ & p.Glu 2059 Glu & $1 / 12(1 / 24)$ & $8 / 50(9 / 100)$ \\
\hline 61 & c. $8245 \mathrm{~T}>\mathrm{G}$ & p.Asp $2610 \mathrm{Glu}$ & $2 / 12(2 / 24)$ & $5 / 50(5 / 100)$ \\
\hline
\end{tabular}

\section{References}

Collod-Beroud G, Boileau C (2002) Marfan syndrome in the third millennium. Eur J Hum Genet 10:673-681

Collod-Beroud G, Bourdelles SL, Ades L, Ala-Kokko L, Booms P, Boxer M, Child C, Comeglio P, De Paepae A, Hyland JC, Holman K, Kaitila I, Loeys B, Matyas G, Nuitinck L, Peltonen L, Rantamaki T, Robinson P, Steinman B, Junien C, Beroud C, Boileau C (2003) Update of the UMD-FBN1 Mutation Database and creation of an FBN1 Polymorphism Database. Hum Mutat 22:199-208

De Paepe A, Devereux RB, Dietz HC, Hennekam RCM, Pyeritz RE (1996) Revised diagnostic criteria for the Marfan syndrome. Am J Med Genet 62:417-426

Faivre L, Gorlin RJ, Wirtz MK, Godfrey M, Dagoneau N, Samples JR, LeMerrer M, Collod-Beroud G, Boileu C, Munnich A, Cormier-Daire V (2003a) In flame fibrillin-1 gene deletion in autosomal dominant Weill-Marchesani syndrome. J Med Genet 40:34-36

Faivre L, Megarbane A, Alswaid A, Zylberberg L, Aldohayan N, Campos-Xavier B, Barq D, Legeai-Mallet L, Bonaventure J, Munnich A, Cormier-Diare V (2003b) Homozygosity mapping of a Weill-Marchesani syndrome locus to chromosome 19p13.3-p13.2. Hum Genet 110:366-370

Ganguly A, Rock MJ, Prockop DJ (1993) Confirmation-sensitive gel electrophoresis for rapid detection of single-base differences in double-strand PCR products and DNA fragments: evidence for solvent-induced bends in DNA heteroduplex. Proc Natl Acad Sci USA 90:10325-10329

Korkko J, Annunen S, Pihlajamaa T, Prockop DJ, Ala-Kokko L (1998) Conformation sensitive gel electrophoresis for simple and accurate detection of mutations: comparison with denaturing gradient gel electrophoresis and nucleotide sequencing. Proc Natl Acad Sci USA 95:1681-1685

Korkko J, Kaitila I, Lonnqvist L, Peltonen L, Ala-Kokko L (2002) Sensitivity of conformational sensitive gel electrophoresis in detecting mutations in and Marfan related conditions syndrome. J Med Genet 39:34-41

Lee B, Godfrey M, Vitale E, Hori H, Mattei MG, Sarfarazi M, Tsipouras P, Ramirez F, Pyeritz RE, Dietx HC (1991) Linkage of Marfan syndrome and a phenotypically related disorder to two different fibrillin genes. Nature 352:330-334

Nagase T, Nakayama M, Nakajima D, Kikuno R, Ohara O (2001) Prediction of the coding sequences of unidentified human genes. $\mathrm{XX}$. The complete sequences of 100 new cDNA clones from brain which code for large proteins in vitro. DNA Res 8:85-95

Wirtz MK, Samples JR, Kramer PL, Rust K, Yount J, Acott TS, Koler RD, Cisler J, Jahed A, Gorlin RJ, Godfrey M (1996) Weill-Marchesani syndrome - possible linkage of the autosomal dominant form to15q21.1. Am J Med Genet 65:68-75 